\title{
双核铑催化的去对称化环异构化反应：构建含炔基取代的 手性氮杂季碳中心的呋喃并二氢哌啶
}

\author{
袁海瑞王剑波* \\ (北京大学化学与分子工程学院 北京分子科学国家研究中心 北京 100871)
}

\section{Constructing Furan-Fused Dihydropiperidines with an Alkyne- Substituted Aza-quaternary Stereocenter via Rh(II)-Catalyzed Desymmetric Cycloisomerization}

\author{
Yuan, Hairui Wang, Jianbo* \\ (Beijing National Laboratory of Molecular Sciences (BNLMS), College of Chemistry and Molecular Engineering, \\ Peking University, Beijing 100871)
}

具有炔基取代的手性中心的分子骨架在药物分子 和天然产物中广泛存在, 主要因为炔基基团会影响药物 分子的亲脂性和刚性等 ${ }^{[1]}$. 另外, 炔基取代的化合物在 有机合成化学中也是一类重要的合成子, 可以通过炔基 官能团的后修饰实现复杂分子的合成. 因此实现炔基取 代的手性分子的合成具有重要意义. 对于该类骨架分子 的合成方法主要有三种策略: (1)利用末端炔烃或者炔基 硅试剂作为炔基化来源, 通过直接不对称炔基化方法构

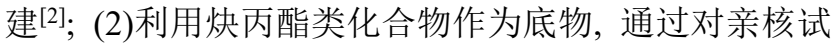
剂进行不对称亲核加成或亲核取代的方法构建 ${ }^{[3]}$; (3)利 用去对称化的策略. 其中, 去对称化的策略可以弥补前 两种合成策略的弊端. 首先, 去对称化的策略对大位阻 的手性季碳中心的构建具有优势. 另外, 利用去对称化 的策略可以不需要考虑反应底物反应活性低的问题, 以 及底物中含有相似基团导致对映选择性差的问题. 然 而, 到目前为止, 使用去对称化的策略实现炔基取代的 手性季碳中心的报道还很少. 在之前的报道中, 去对称 化的策略主要是利用金催化的二炔氢氨化策略 ${ }^{[4]}$ 以及利 用一价铑催化的 $[2+2+2]^{2}$ 环加成反应 ${ }^{[5]}$, 但是存在对映 选择性较差和底物局限等问题. 因此, 利用去对称化策 略实现炔基取代的季碳中心化合物的高对映选择性合 成仍然具有挑战.

近年来，过渡金属催化二炔化合物的环异构化已经 成为碳环和杂环化合物合成的重要策略, 其中 1,6-二炔
化合物作为一种重要的二炔类型，其环异构化反应更是 引起了广泛关注. 对于 1,6-二炔化合物的不对称分子内 环异构化反应目前为止尚未报道. 因此，探索新的策略 来实现 1,6-二炔的对映选择性环异构化具有重要意义, 这也是构建手性环状分子的有效途径. 近年来，华南理 工大学祝诗发课题组 ${ }^{[6-7]}$ 在炔烃化学方面进行了深入地 研究, 尤其是最近发展了双核铑催化炔醛不对称转化的 体系, 为 1,6-二炔的对映选择性环异构化提供了思路.

2021 年, 华南理工大学化学与化工学院祝诗发课 题组和中南大学陈凯博士合作, 在 1,6-二炔的对映选择 性环异构化领域取得了突破性进展，他们发展了一种双 核铑催化的新型二炔去对称化环异构化策略，一步合成 了两个杂环和一个炔基取代的手性氮杂季碳中心，为炔 基取代手性氮杂季碳中心和呋喃并二氢哌啶衍生物的 高效构建提供了方法 ${ }^{[8]}$.

通过尝试不同的金属催化剂、不同的手性双核铑催 化剂、溶剂、浓度和温度，他们最终确定以 $1 \mathrm{~mol} \%$ $\mathrm{Rh}_{2}(R \text {-PTAD })_{4}$ 作为催化剂, 1,2 -二氯乙烷 $(0.1 \mathrm{~mol} / \mathrm{L})$ 作为 溶剂, $25{ }^{\circ} \mathrm{C}$ 反应 $48 \mathrm{~h}$ 作为标准反应条件(Scheme 1). 该 反应具有很好的底物普适性，不同的烷基、硅基、芳基 取代的底物均能以较好的收率和对映选择性得到相应 的去对称化环异构化的产物. 醚类的二炔作为底物时, 反应可以中等的产率和对映选择性得到呋喃并二氢吡 喃的产物. 同时，该作者通过理论计算和控制实验对

* Corresponding author. E-mail: wangjb@pku.edu.cn. Published online November 22, 2021. 

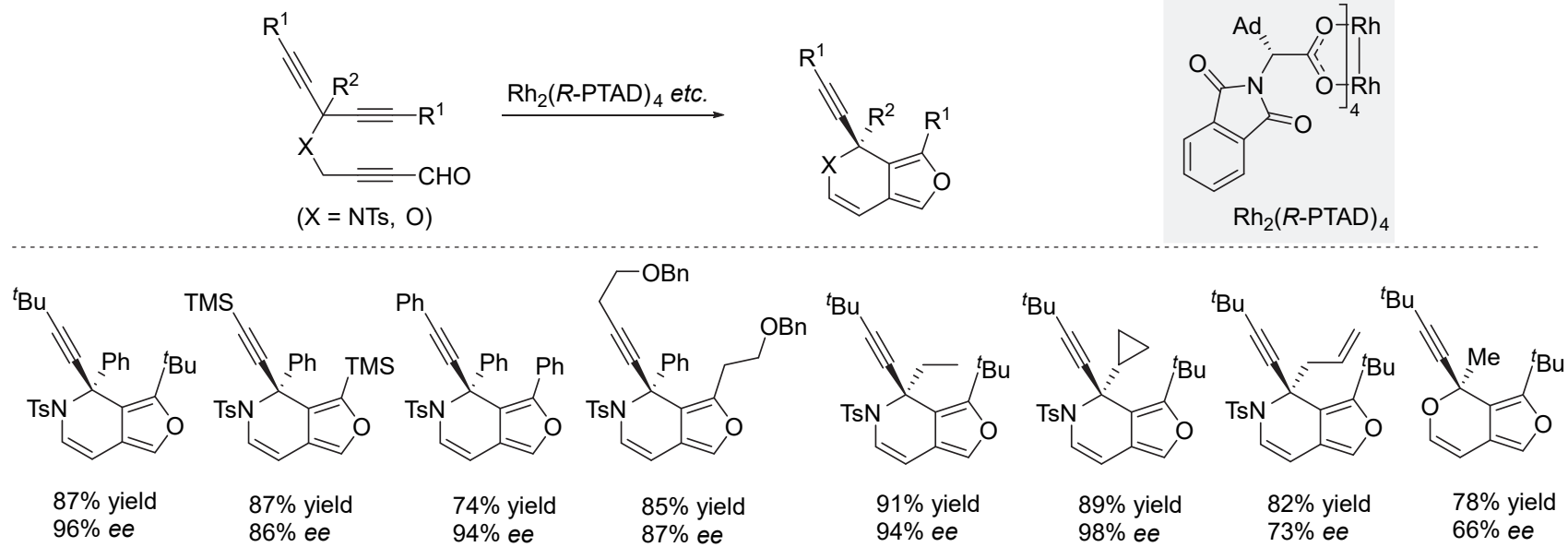

图式 1 对映选择性的去对称环化异构化反应的底物普适性

Scheme 1 Substrate scope of enantioselective desymmetric cycloisomerization

这一反应的机理进行了深入研究. 密度泛函理论(DFT) 计算结果表明, 该去对称化环异构化过程经历了 $[3+2]$ 环化过程, 得到了铑卡宾中间体. 由于 $[3+2]$ 过程形成 了芳香性的呋喃环, 能垒低, 释放能量大, 该过程是动 力学和热力学均有利的过程. 铑卡宾中间体随后发生的 1,2-氢迁移, 同样是热力学非常有利的过程. 作者也对 其它可能的路径进行了计算, 但均不及 $[3+2]$ 环化/1,2氢迁移过程有利. 另外, 通过对过渡态分析发现, 与炔 基相连的亚甲基和醛基上的氢与羧酸铑配体中氧原子 的距离均小于氧原子和氢原子的范德华半径之和 0.272 $\mathrm{nm}$, 说明可能存在非经典的多重弱氢键作用. 通过氛代 控制实验和对照实验证明, 反应确实经历了分子内 $1,2-$ 氢迁移过程以及醛基在该反应的必要性.

另一方面，该作者分离了苄基取代的炔醛底物和特 戊酸铑二聚体形成的配合物晶体. 通过晶体结构分析炔 基和 $\mathrm{Rh}(\mathrm{II})$ 进行的配位, 发现其与以往报道的炔醛通过 羰基氧和铑进行的配位有所不同. 对所得晶体的晶体数 据和核磁数据进行分析发现, 双核铑和底物之间属于弱 配位. 此外, 晶体中底物炔基旁的亚甲基和醛基的氢原 子与催化剂羧酸配体的氧原子之间的距离表明, 它们之 间存在着三重弱氢键. 这样的弱配位配合物之所以能够 在溶剂挥发条件下分离得到, 多重弱氢键可能起到了重 要作用. 另外, 这些弱氢键的存在也在一定程度上解释 了独特的螺旋桨型双核铑催化剂在该反应中所表现出 的独特的催化性能. 对手性铑 $\mathrm{Rh}_{2}(R-\mathrm{PTAD})_{4}$ 和底物结 合的络合物晶体进行分析, 发现由于金刚烷基的位阻原 因, 只有单炔醛与铑配位, 而另一个位阻更大的配位点 被体积更小的乙酸乙酯占据. 结合所得晶体的结构以及 通过对反应的手性控制进行计算分析, 发现炔基末端的 取代基位阻对反应的对映选择性有重要影响. 炔基上的 取代基越大, 两种构型的能量差也越大, 反应的对映选

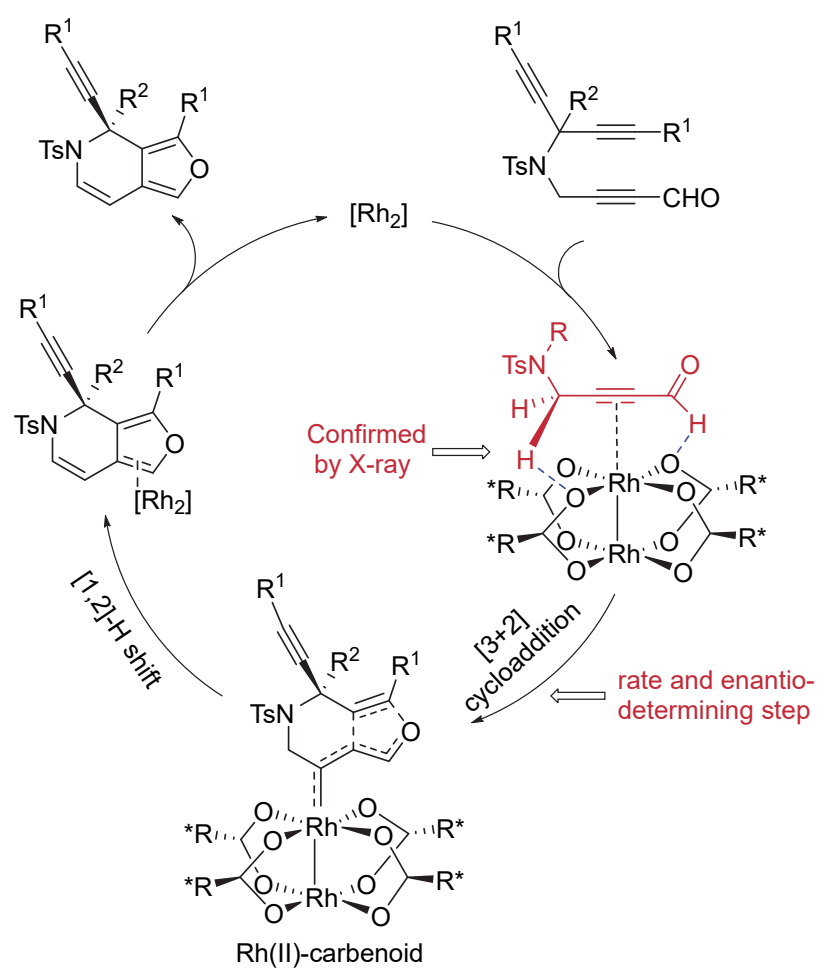

图式 $2 \mathrm{Rh}(\mathrm{II})$ 催化去对称环化异构化的反应机理

Scheme 2 Reaction mechanism of enantioselective Rh(II)catalyzed desymmetric cycloisomerization

择性越好, 这和从底物拓展实验反应中观察到的结果一 致. 该项研究实现了 $\mathrm{Rh}(\mathrm{II})$ 催化 1,6-二炔化合物的对映 选择性去对称环异构化反应, 并分离得到炔- $\mathrm{Rh}(\mathrm{II})$ 的 弱 $\pi$-配合物晶体，有助于 $\mathrm{Rh}(\mathrm{II})$ 催化炔烃对映选择性转 化机理的进一步理解.

\section{References}

[1] Corbett, J. W.; Ko, S. S.; Rodgers, J. D.; Gearhart, L. A.; Magnus, N. A.; Bacheler, L. T.; Diamond, S.; Jeffrey, S.; Klabe, R. M.; Cordova, B. C.; Garber, S.; Logue, K.; Trainor, G. L.; Anderson, P. S.; 
Erickson-Viitanen, S. K. J. Med. Chem. 2000, 43, 2019.

[2] Li, Z. L.; Fang, G. C.; Gu, Q. S.; Liu, X. Y. Chem. Soc. Rev. 2020, $49,32$.

[3] Dai, J.; Wang, Z.; Deng, Y.; Zhu, L.; Peng, F.; Lan, Y.; Shao, Z. Nat. Commun. 2019, 10, 5182.

[4] Mourad, A. K.; Leutzow, J.; Czekelius, C. Angew. Chem., Int. Ed. 2012, 51,11149 .

[5] Tanaka, K.; Osaka, T.; Noguchi, K.; Hirano, M. Org. Lett. 2007, 9 ,
1307.

[6] Wu, R.; Chen, K.; Ma, J.; Yu, Z.; Zhu, S. Sci. China Chem. 2020, 63, 1230.

[7] Liu, X.; Xu, X.; Hu, W. Chin. J. Org. Chem. 2020, 40, 4370 (in Chinese). (刘向荣, 徐新芳, 胡文浩, 有机化学, 2020, 40, 4370.)

[8] Wu, R.; Lu, J.; Cao, T.; Ma, J.; Chen, K.; Zhu, S. J. Am. Chem. Soc. 2021, 143, 14916. 